\title{
PREDICTION OF RESIDUAL RESISTANCE OF A TRIMARAN VESSEL BY USING AN ARTIFICIAL NEURAL NETWORK
}

\author{
UDC 629.5.022.3:629.5.015.2
}

Original scientific paper

\section{Summary}

Trimaran hull forms have been popular recently in both commercial and military usage due to reduction in resistance at high speeds, better stability, and greater deck area compared to conventional monohull vessels. Determination of the location of the side hulls is most critical to get higher hydrodynamic performance. Therefore, many studies in the literature are related to defining the location of the side hulls for trimaran vessels. Most of the studies have been carried out experimentally or numerically. In this study, an artificial neural network (ANN) model was used to predict the residual resistance coefficient of a trimaran model. The model uses four parameters which are the transverse and longitudinal positions of the side hulls, the longitudinal centre of buoyancy and the Froude number to predict the residual resistance of the trimaran model. The experimental data of the trimaran model were used to train the neural network model in order to develop a more reliable model. Several neural network models were developed and tested to find the one with minimum error. The study showed that the residual resistance coefficients of the trimaran model were predicted with high accuracy levels compared to the model experimental data. It was also shown that an ANN is a useful alternative method to model tests and numerical simulations. The developed model can be used to reduce the number of model tests or numerical simulations as well as to obtain the optimum location of the side hulls in terms of resistance.

Key words: $\quad$ trimaran; residual resistance; side hull; artificial neural network

\section{Introduction}

Trimaran hull form usage, especially for high-speed naval vessels, passenger ferries and pleasure crafts, has recently increased due to reduction in resistance at high speeds. Trimarans have slender hull forms which means less wave-making resistance, and they offer high stability and seakeeping performance in addition to great deck area.

The side hull location of trimarans may increase or decrease resistance due to the interaction between the generated waves from the main hull and the side hulls. Therefore, determination of the positioning of the side hulls should be investigated in detail in order to reduce resistance. Furthermore, the increase in demand for trimaran vessels leads researchers to study the effect of side hull positioning on trimaran hydrodynamics, motion characteristics and manoeuvrability. Most of the studies for trimaran vessels comprise experimental or 
numerical investigation. Zhang [1] carried out experimental and theoretical studies to investigate the resistance, seakeeping performance and manoeuvrability of a trimaran fast ferry. The effect of side hull configurations on the performance of the trimaran vessel was presented. Larsson et al. [2] used numerical methods to investigate the effect of side hull configurations on the hydrodynamics of a trimaran ship. Yang et al. [3] used four different methods based on Euler's equation and linear potential flow method to determine the optimum location of the side hulls of a trimaran vessel with decreased wave resistance. Degiuli et al. [4] used different methods of experimental determination of wave pattern resistance for a trimaran model based on wave height measurements in several longitudinal measurement cuts. Theoretical predictions of wave resistance were also obtained and a comparison between theoretical predictions and experimentally determined wave pattern resistance showed poor agreement. Doctors and Sahoo [5] compared the generated waves of a trimaran by using a theoretical method for different side hull positions, and the results were compared with experimental results. Mynard et al. [6] used numerical simulations, slender body theory and experiments to calculate the wave resistance of a trimaran hull form with various side hull positions. Mahmood and De Bo [7] carried out numerical simulations in order to obtain the fluid flow of a trimaran ship by using commercial software. The total resistance values were calculated for various Froude numbers and they were validated with model tests. Son et al. [8] carried out numerical simulations of a high-speed trimaran vessel with a round bilge hull to predict the total resistance value. Three different hull forms for the centre hull were analyzed and the results of the numerical method were compared with the experimental data. The interference effect between the hulls of multihulls is critical to obtain the optimum side hull positioning. Most of the studies related to the resistance of multihulls focus on investigation of the interference effect. Battistin [9] carried out experiments for sixteen different trimaran configurations using analytical and numerical methods. The importance of wave interference on the trimaran resistance was highlighted. Mizine et al. [10] performed numerical calculations for a trimaran ship in order to understand the factors leading to the interference effect, and the results were compared to model test data. Zaghi et al. [11] performed a combined experimental and numerical study that focused on the effect of the separation distance between the hulls for a high-speed catamaran. Farkas et al. [12] carried out a numerical investigation of the interference phenomenon for a series 60 catamaran. The influence of forward speed and separation on interference was investigated using Computational Fluid Dynamics (CFD). Yildiz et al. [13] carried out numerical and experimental work to predict the total resistance and residual resistance of a trimaran for nine side hull configurations and the interference factors were calculated to determine the optimum side hull location. Duman et al. [14] carried out numerical analyses of a trimaran to investigate the interference effect between the main hull and side hulls. The interference effect was calculated by using the total resistance, residual resistance and wave resistance. All of these studies include experimental or numerical calculations to analyze the effect of the side hull locations on trimaran resistance. The residual resistance, wave resistance and interference effect results were analyzed to see the effect of the different layouts on the trimaran resistance. The researchers not only focused on the resistance of trimaran vessels but also on their stability, motion and manoeuvring characteristics. Khoob et al. [15] investigated the effect of the side hull locations on wave-induced motions and load responses for a trimaran ship using statistical short-term analysis. Zhang et al. [16] studied the roll motion of a trimaran vessel for different side hull configurations and showed the importance of the side hull locations on its dynamic stability. Gong et al. [17] used a hybrid numerical method to simulate a trimaran vessel with different layouts moving forward in waves, and the motion response and added resistance were calculated. Jiang et al. [18] carried out manoeuvring tests of a trimaran model with different layouts and the effect of the different positions of the side hulls on the manoeuvrability parameters were analyzed. 
Experiments give the most reliable and accurate results. However, carrying out an experiment is time-consuming and expensive. An alternative to the experiment is numerical simulations using CFD which is also time-consuming, unless you have high capacity workstations to reduce the computational time, but this means extra cost.

ANNs are another way to predict ship resistance, which are fast and cost-effective compared to experiments and numerical methods. The published papers of recent years show that ANNs are a useful tool for ship resistance prediction. For example; Origosa et al. [19] presented a neural network model to predict the form factor and wave resistance coefficient of ships by using the data of hull geometry coefficients and the forward speed. The results were compared with the Holtrop \& Mennen method [20] and the proposed model gave accurate results. Grabowska and Szczuko [21] predicted the residual resistance coefficient of off-shore vessels by using an ANN with various training algorithms. The results were compared with model tests and good correlation was observed between the results. They also proposed new input parameters to improve the accuracy of the developed network. Cepowski [22] used an ANN to develop a function for the prediction of the added wave resistance of a ship. The experimental results of 14 ships were used to train the neural network model. Different neural network types were implemented and the best one with the minimum error used a multi-layer perceptron (MLP) algorithm. The developed function was also used to predict the resistance of ships which were not used in the training of the network, and the results were compared with experimental results to check its accuracy. The predicted results were compatible with the model test results. Yang et al. [23] used the Radial Basis Function neural network (RBFNN) to calculate the resistance of a 13500TEU container ship at different drafts. The results were compared with four different machine learning models and verified with experimental data. It was concluded that the prediction results of the RBFNN are more accurate. Kim et al. [24] proposed a predictor model to estimate the resistance of ice-going ships based on an ANN. Model test results and full-scale measurements were used to train six different ANN models. The developed model results were compared to the full-scale test data and semi-empirical formulas.

There are also papers in the literature related to trimaran resistance prediction by using ANNs. Royce et al. [25] developed four different ANN models by using experimental results of trimaran hulls and predicted the trimaran residuary resistance, trim and sinkage. The results were obtained for three different side hull locations which were not considered in training the neural network. The results were compared with test results and good consistency was observed. Muk-Pavic et al. [26] developed a neural network model which was trained by using model tests to calculate the total resistance for a trimaran hull form, Tri-SWACH. The results showed the ANN's potential to be a resistance prediction tool for Tri-SWACH preliminary design.

This study seeks to develop a neural network model to predict the residual resistance coefficient of a trimaran hull for different side hull locations at various forward speeds. The neural network model predicts the resistance by use of trimaran parameters such as transverse position and longitudinal position of the side hulls, longitudinal centre of buoyancy and forward speed. The trimaran model test results were used to develop a reliable model and the neural network model prediction results showed good agreement when compared with the experimental results.

\section{Experimental data}

The trimaran model used in this study is a 1/125 scale high-speed displacement frigatetype. The hull line plans and main dimensions of the model trimaran are presented in Figure 1 and Table 1, respectively. 
Table 1 Trimaran model characteristics

\begin{tabular}{lll}
\hline & Center Hull & Side Hull \\
\hline$\Delta(\mathrm{kg})$ & 15.52 & 0.48 \\
$L_{O A}(\mathrm{~m})$ & 2.14 & 0.61 \\
$L_{W L}(\mathrm{~m})$ & 2.10 & 0.61 \\
$B_{M}(\mathrm{~m})$ & 0.21 & 0.03 \\
$B_{W L}(\mathrm{~m})$ & 0.19 & 0.03 \\
$T(m)$ & 0.07 & 0.06 \\
$L C B(m)$ & 0.97 & 0.31 \\
Static Wetted Area $\left(\mathrm{m}^{2}\right)$ & 0.48 & 0.06 \\
\hline
\end{tabular}

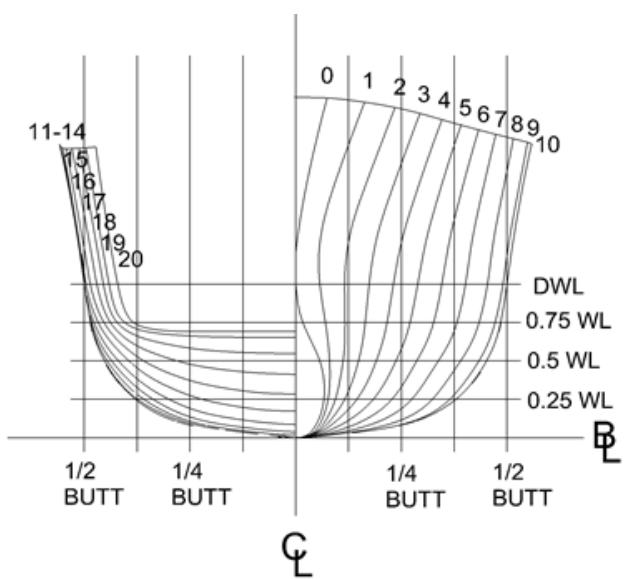

\section{MAIN HULL}

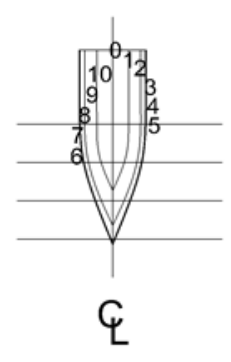

SIDE HULL

Fig. 1 Plan of the trimaran hull lines

Nine different configurations were selected to investigate the effect of the longitudinal and transverse location of the side hulls. The longitudinal and transverse positions of the outriggers are given in Table 2. The longitudinal positions are defined as $s^{\prime}$ and the transverse positions are defined as $t^{\prime}$ (Figure 2).

Table 2 Trimaran side hull configurations

\begin{tabular}{ccc}
\hline CONFIGURATION & $s^{\prime}=s / L$ & $t^{\prime}=t / B$ \\
\hline A & 0.28 & 0.86 \\
B & 0.23 & 0.86 \\
C & 0.18 & 0.86 \\
D & 0.28 & 1.07 \\
E & 0.23 & 1.07 \\
F & 0.18 & 1.07 \\
G & 0.28 & 1.27 \\
H & 0.23 & 1.27 \\
I & 0.18 & 1.27 \\
\hline
\end{tabular}

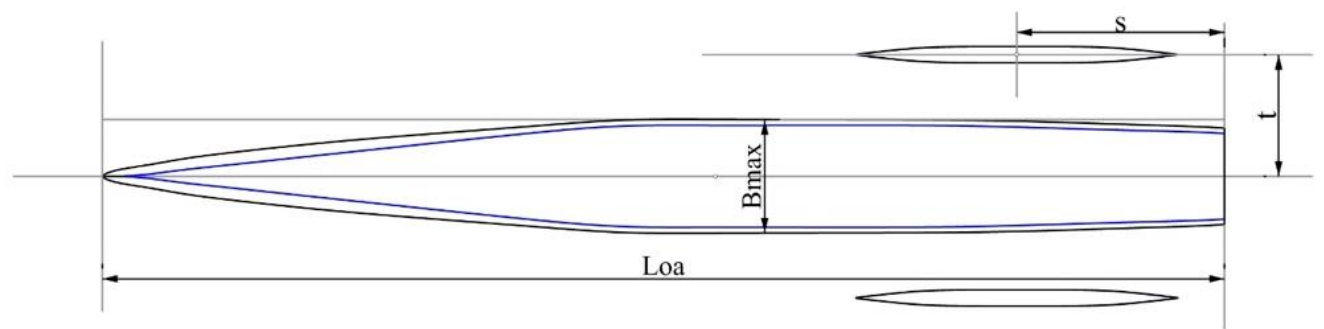

Fig. 2 Trimaran transverse and longitudinal positions

The experimental data used in this study was obtained by Carr and Dvorak [27] who carried out towing tank tests to calculate the residual resistance data of the trimaran hull for 
each configuration at various forward speeds for the range of Froude numbers from 0.10 to 0.50. Total resistance was obtained from the experiments and the residual resistance coefficient was calculated. The total resistance can be divided into residual resistance and friction resistance.

$$
R_{T}=R_{F}+R_{R}
$$

The total resistance coefficient is calculated as:

$$
C_{T}=\frac{R_{T}}{\frac{1}{2} \rho S V^{2}}
$$

where $\rho$ is the fluid density, $S$ is the hull wetted surface area and $V$ is the ship speed.

As stated in equations (3) and (4), the friction resistance and friction resistance coefficient were calculated by using the ITTC friction line [28].

$$
\begin{gathered}
R_{F}=C_{F} \frac{1}{2} \rho S V^{2} \\
C_{F}=\frac{0.075}{(\log R e-2)^{2}}
\end{gathered}
$$

Finally, the residual resistance coefficient can be calculated as:

$$
C_{R}=C_{T}-C_{F}
$$

\section{Artificial neural networks}

An ANN is an information processing method based on a biological nervous system that consists of neurons and their connections. Neurons are connected in several layers, interconnected by inputs and outputs. Figure 3 shows the model of a neuron which forms the basis for designing ANNs. A neural network has an input layer, hidden layers and an output layer. Each input $\left(x_{i}\right)$ passes through the hidden layers with a specific weight $\left(w_{i}\right)$. The output $(y)$ of each neuron is the sum of each input added via bias $(b)$ and transfer function $(f)$ as follows:

$$
y=f_{j}\left(\sum_{i=1}^{n} w_{i j} x_{i}+b_{j}\right)
$$




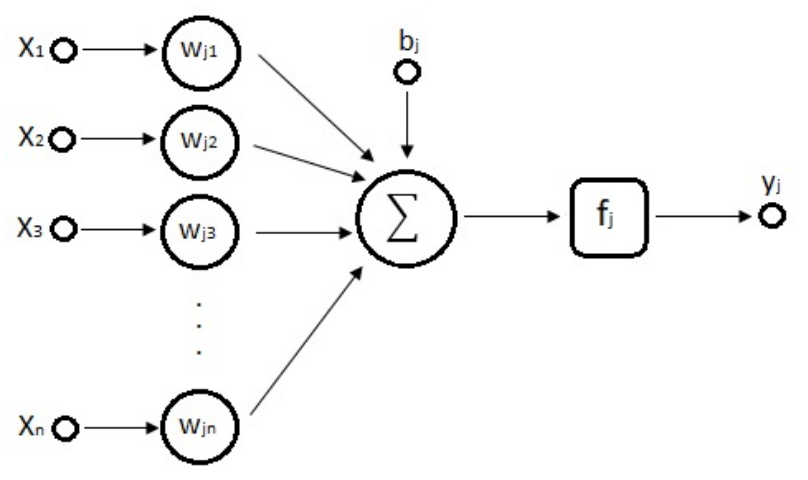

Fig. 3 Nonlinear model of a neuron [29]

There are three activation functions often used in the hidden layer and the output layer. These functions are purelin, standard logistic function and hyperbolic tangent.

Different neural network types such as Generalized Regression Neural Network (GRNN), Multi-layer Perceptron (MLP), Radial Basis Function, Support Vector Machines (SVM) can be used in resistance prediction problems. Multi-layer perceptron (MLP), which is the most widely used neural network type [29],[30], was used in this study for the prediction of the residual resistance coefficients of trimaran vessels. A multi-layer perceptron, which is a feedforward artificial network, with a backpropagation algorithm was applied. The structure of the MLPs can be seen in Figure 3.

Determination of neural network topology is essential to develop a simple and reliable model. The number of hidden neurons and the training and learning algorithms should be decided carefully. Backpropagation algorithms are used for the training of multi-layer perceptron networks. In this study, Levenberg-Marquardt (LM), which is a type of quasiNewton algorithm, and the Scaled Conjugate Gradient (SCG) algorithm were used. In addition, Bayesian regularization (BR) was also applied which does not need a validation data set. BR offers a great advantage when the number of data for training is limited [31]. The parameters of the used training functions were taken as default values as shown in MATLAB Neural Network Toolbox [32]. The default values used for the training parameters are listed in Table 3.

Table 3 Training parameters' values used for the neural network models

\begin{tabular}{|l|l|}
\hline Training Parameters & Values \\
\hline Maximum number of epochs to train & 1000 \\
\hline Performance goal & 0 \\
\hline Maximum validation failures & 100 \\
\hline Minimum performance gradient & $1 \mathrm{e}-7$ \\
\hline Initial $\mu$ & 0.001 \\
\hline Decrease factor for $\mu$ & 0.1 \\
\hline Increase factor for $\mu$ & 10 \\
\hline Maximum value for $\mu$ & $1 \mathrm{e}+10$ \\
\hline Epochs between displays & 25 \\
\hline Maximum time to train in seconds & inf \\
\hline
\end{tabular}

The mean squared error (MSE) values were obtained to evaluate the accuracy of the neural network as follows: 


$$
M S E=\frac{1}{n} \sum_{i=1}^{n}\left(\rho_{a}-\rho_{e}\right)^{2}
$$

where $n$ is the number of available data, $\rho_{a}$ is the actual data (target) and $\rho_{e}$ is the output data.

\section{Results and discussion}

MATLAB Neural Network software [32] was used for the training and testing of the ANNs. The Froude number, $L C B$, and the longitudinal and transverse locations were used as the input data, and the residual resistance coefficient $\left(C_{R}\right)$ was obtained as the output data. The $L C B$ was also made non-dimensional as the divided length of the hull $\left(L C B / L_{W L}\right)$ since the remaining input parameters (Froude number, $s^{\prime}, t^{\prime}$ ) are non-dimensional. The range of input and output data is between 0 and 1 so there is no need to apply an extra normalization.

The experimental data belonging to the 8 different configurations of the side hulls used for the network development was divided into training, validation and test sets. The training data was used to train the model by adjusting the weights, the validation data set was used to avoid overfitting and to tune the weights, and the test data was used to test the accuracy of the ANN predictions.

In this study, the provided experimental data for the 8 different configurations included 192 cases in total which means 24 cases for each configuration. The dataset was randomly divided into training, validation and test datasets which comprise 134, 28 and 30 cases, respectively. It means that $70 \%$ of the total data was used for the training, $15 \%$ for validation and $15 \%$ for testing. Only configuration $F$ was excluded for the case study and it was not used in the development of the neural network model.

In order to obtain the most accurate neural network model which offers the lowest testing error, several models were developed with various numbers of neurons in the single hidden layer, and with different training and transfer functions. MSE errors of train, test and validation data sets for each tested model are shown in Table 4. The structure of the developed ANN models is shown in Figure 4.

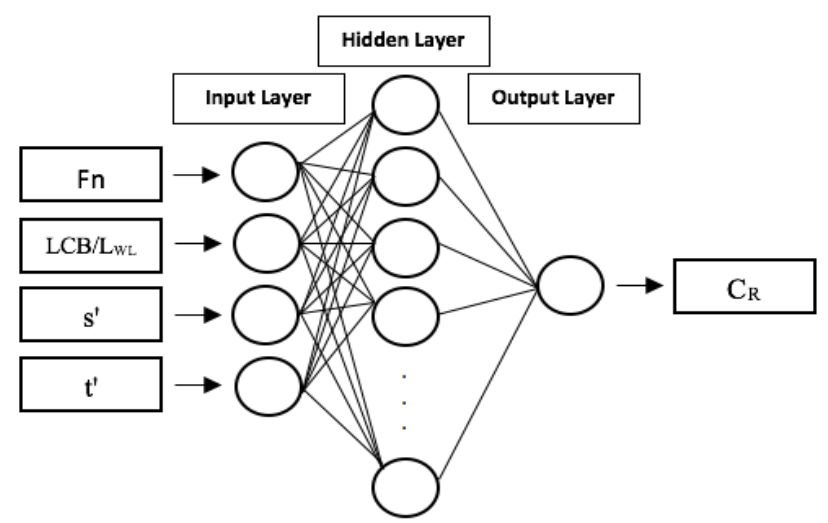

Fig. 4 Structure of the developed ANN model 
Table 4 The tested neural networks with MSE values

\begin{tabular}{|c|c|c|c|c|c|c|}
\hline No. & $\begin{array}{l}\text { Number } \\
\text { of } \\
\text { hidden } \\
\text { neurons }\end{array}$ & $\begin{array}{l}\text { Training } \\
\text { function }\end{array}$ & $\begin{array}{l}\text { Transfer } \\
\text { function }\end{array}$ & Train & Test & Validation \\
\hline 1 & 1 & trainlm & tansig-tansig & 0.0484 & 0.0491 & 0.0478 \\
\hline 2 & 2 & trainlm & tansig-tansig & 0.0510 & 0.0729 & 0.0421 \\
\hline 3 & 3 & trainlm & tansig-tansig & 0.0199 & 0.0212 & 0.0206 \\
\hline 4 & 3 & trainlm & tansig-purelin & 0.0491 & 0.0509 & 0.0471 \\
\hline 5 & 4 & trainlm & tansig-tansig & 0.0257 & 0.0348 & 0.0225 \\
\hline 6 & 4 & trainlm & tansig-purelin & 0.0292 & 0.0307 & 0.0310 \\
\hline 7 & 5 & trainlm & tansig-tansig & 0.0259 & 0.0185 & 0.0152 \\
\hline 8 & 5 & trainlm & tansig-purelin & 0.0206 & 0.0182 & 0.0205 \\
\hline 9 & 5 & trainlm & logsig-purelin & 0.0343 & 0.0433 & 0.0471 \\
\hline $10^{*}$ & 6 & trainlm & tansig-purelin & 0.0200 & 0.0154 & 0.0132 \\
\hline 11 & 7 & trainlm & tansig-purelin & 0.0356 & 0.0371 & 0.0477 \\
\hline 12 & 8 & trainlm & tansig-purelin & 0.0191 & 0.0214 & 0.0209 \\
\hline 13 & 9 & trainlm & tansig-purelin & 0.0193 & 0.0198 & 0.0210 \\
\hline 14 & 10 & trainlm & tansig-purelin & 0.0189 & 0.0376 & 0.0401 \\
\hline 15 & 6 & trainlm & tansig-tansig & 0.0145 & 0.0347 & 0.0235 \\
\hline 16 & 6 & trainlm & tansig-logsig & 0.1020 & 0.1636 & 0.1071 \\
\hline 17 & 6 & trainlm & logsig-logsig & 0.1066 & 0.1901 & 0.0811 \\
\hline 18 & 6 & trainscg & logsig-tansig & 0.1300 & 0.1067 & 0.0749 \\
\hline 19 & 6 & trainscg & tansig-purelin & 0.0297 & 0.0454 & 0.0241 \\
\hline 20 & 6 & trainlm & purelin-purelin & 0.0449 & 0.0384 & 0.0701 \\
\hline 21 & 15 & trainlm & purelin-purelin & 0.0494 & 0.0552 & 0.0318 \\
\hline 22 & 6 & trainbr & tansig-purelin & 0.0366 & 0.0338 & $\mathrm{NaN}$ \\
\hline 23 & 10 & trainbr & tansig-purelin & 0.0379 & 0.0311 & $\mathrm{NaN}$ \\
\hline $24^{*}$ & 15 & trainbr & tansig-purelin & 0.0122 & 0.0131 & $\mathrm{NaN}$ \\
\hline 25 & 10 & trainbr & logsig-purelin & 0.0135 & 0.0153 & $\mathrm{NaN}$ \\
\hline 26 & 15 & trainbr & logsig-purelin & 0.0082 & 0.0430 & $\mathrm{NaN}$ \\
\hline
\end{tabular}

*refers to the best ANN model

The tested neural network with the lowest test MSE value is referred to as the most accurate neural network model. As can be seen from Table 4, the minimum test error was obtained in tests 10 and 24 with a test error of 0.0154 and 0.0131 , respectively. The first network (number 10) with the lowest test error has 6 hidden neurons in the single hidden layer, and the LM algorithm was used for the training function. The activation functions for the hidden layer and output layer are tansig and purelin, respectively. It was also observed that the best ANN model with the lowest test error has the lowest validation error. However, it is not valid for the training errors and the best ANN model does not give the lowest training error. The lowest training error with the LM algorithm belongs to test number 15 which is 0.0145 and the test 
error of this network is 0.0347 , which can result in the overfitting problem. Table 4 also shows that increasing the number of hidden neurons to more than 6 with the LM algorithm did not improve the performance of the neural network model. The second network (number 24) with the lowest test error uses BR as the training function and has 15 hidden neurons in the single hidden layer. The activation functions for the hidden layer and the output layer are tansig and purelin, respectively.

Both network models showed similar output values. Therefore, the ANN model with LM algorithm (number 10) was used in the rest of the study as the best neural network as it offers the fastest training function. Figure 5 shows the performance plot of the best neural network model. It shows that the developed neural network model performed 214 iterations in total and the best validation performance was achieved at the 114th iteration.

The regression graphic between the predicted neural network values and experimental values is shown in Figure 6. The correlation coefficients $(R)$ were obtained as $0.97335,0.90383$, 0.97259 and 0.96637 for training, validation, test and all data-sets, respectively. It can be said that the developed model results are consistent with the experimental results while the $R$ values are very close to 1.0 .

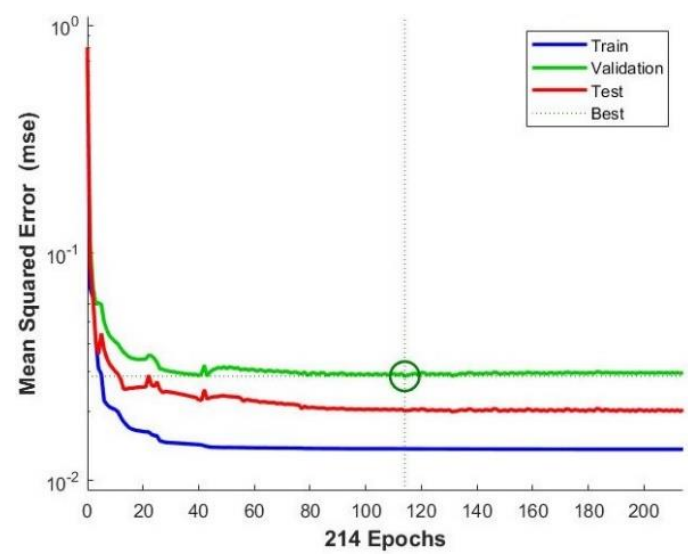

Fig. 5 Performance graphic for the most accurate neural network model
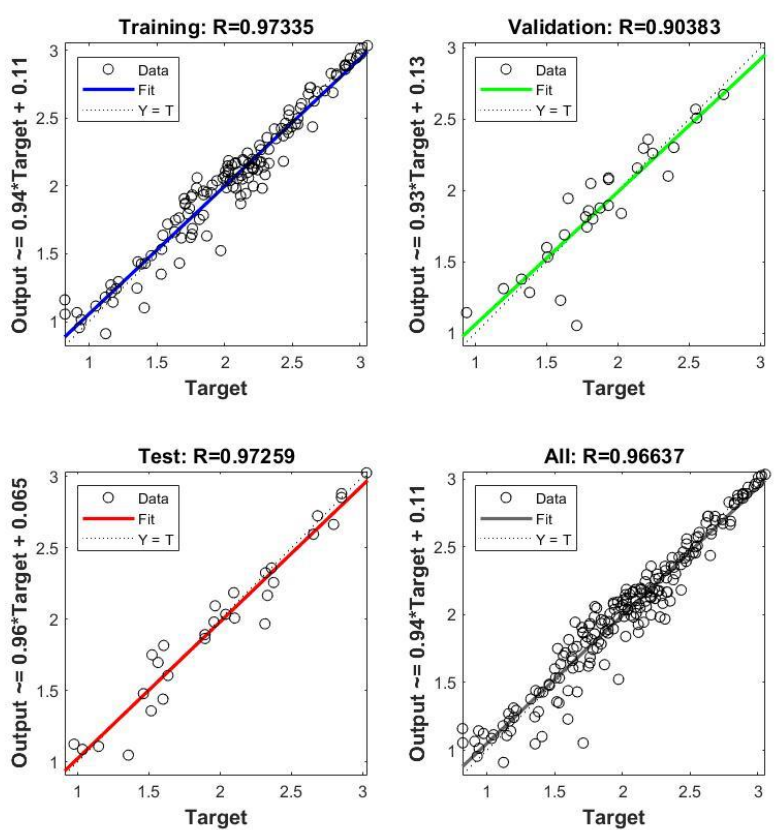

Fig. 6 Regression graphics for the most accurate neural network model 
Figure 7 shows the distribution of residuals between desired and ANN outputs for the best neural network model. In this figure, the residual or error is calculated as the difference between the predicted values and experimental values. The distribution of the error bars near the zero error line (orange line) indicates the more accurate predictions. It can be seen that the largest distribution of error is between -0.2 and 0.2 .

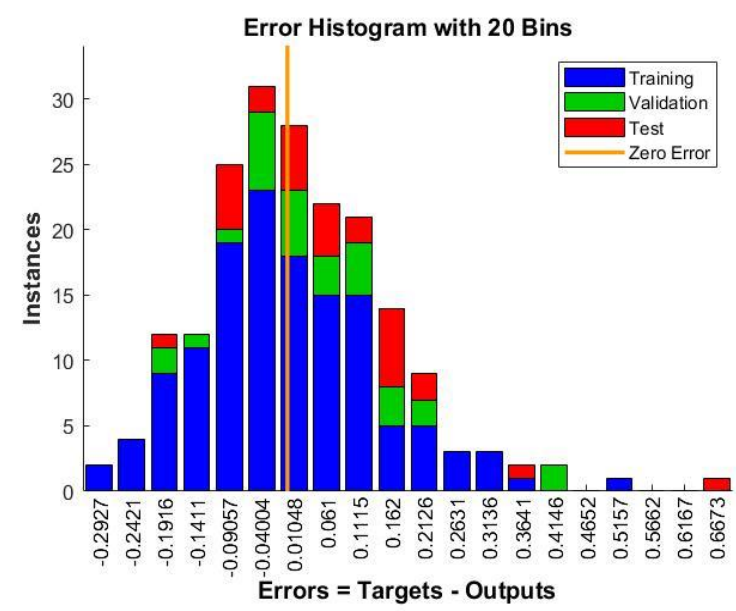

Fig. 7 Distribution of residuals between desired and ANN outputs

The results of the developed network predictions are presented in Figure 8. Figure 8 compares the residual resistance coefficient data from the experiments which were used to develop the network model, with the neural network predictions. The results of the comparison are given separately for each side hull configuration. The results show that the neural network predictions at every speed for all the configurations are in good agreement with the experimental results in general. The nonlinear nature of the experimental results can be seen from the figure and the developed neural network model captured it accurately. The actual results showed rapid changes, especially in the range of Froude numbers between 0.10 and 0.25 , which were not observed in the predictions. The reason for the lack of neural network predictions in this region is the large variation of experimental data in this Froude number range. This could prohibit the neural network from appropriately developing the input-target relationship during training [21]. The number of data used in the network should be increased in this region to capture these rapid changes. The actual values in the Froude number range between 0.25 and 0.50 are smoother and the neural network model predictions coincide with the experimental results in this region. It is important that the developed neural network accurately predicted the main hump region in all the configurations and captured the trend of the curve. 

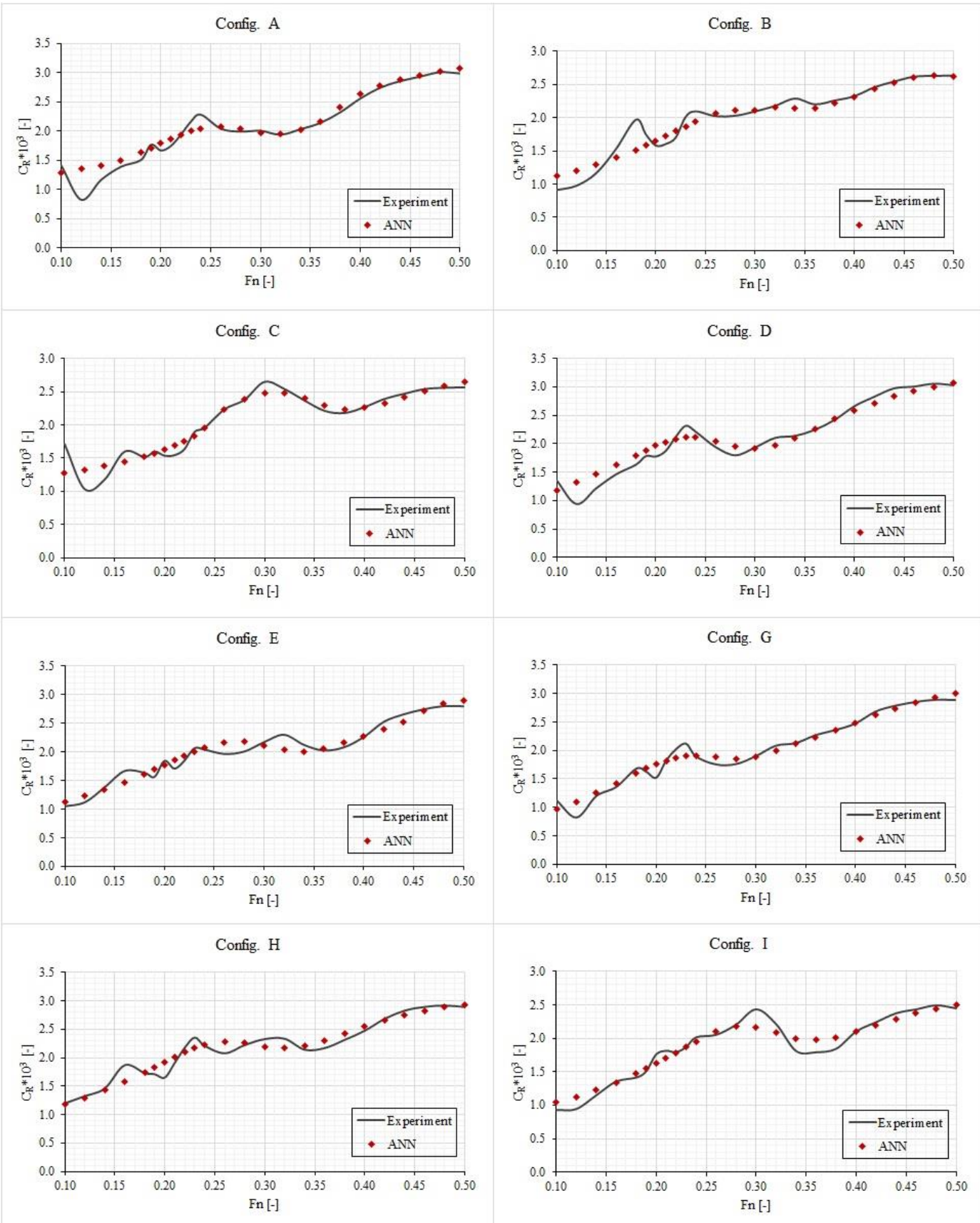

Fig. 8 Comparison of residual resistance coefficients between experimental results and ANN predictions

\section{Case study}

A case study was carried out to check the accuracy of the developed neural network. In the case study, a configuration, whose data was not used for the development of the neural network, was chosen. This means that the data of the case configuration was not previously trained or tested in the developed neural network. Configuration $F$ was chosen for the case study whose longitudinal position is 0.18 and transverse position is 1.07 . The residual resistance coefficients of Configuration $F$ were calculated for a range of Froude numbers between 0.10 and 0.50 by using the developed network model.

The results of the case study are shown in Figure 9. To show the accuracy of the neural network model, the results were compared with the experimental data. Figure 9 shows that the neural network predicted the data (which was not used for the development of the network) at an acceptable level. The experimental results show rapid changes over the whole Froude number 
range, while the ANN results have a smooth line that captures the trend of the experimental data. It is important that the neural network accurately captured the main hump region between the Froude numbers 0.25 and 0.35 .

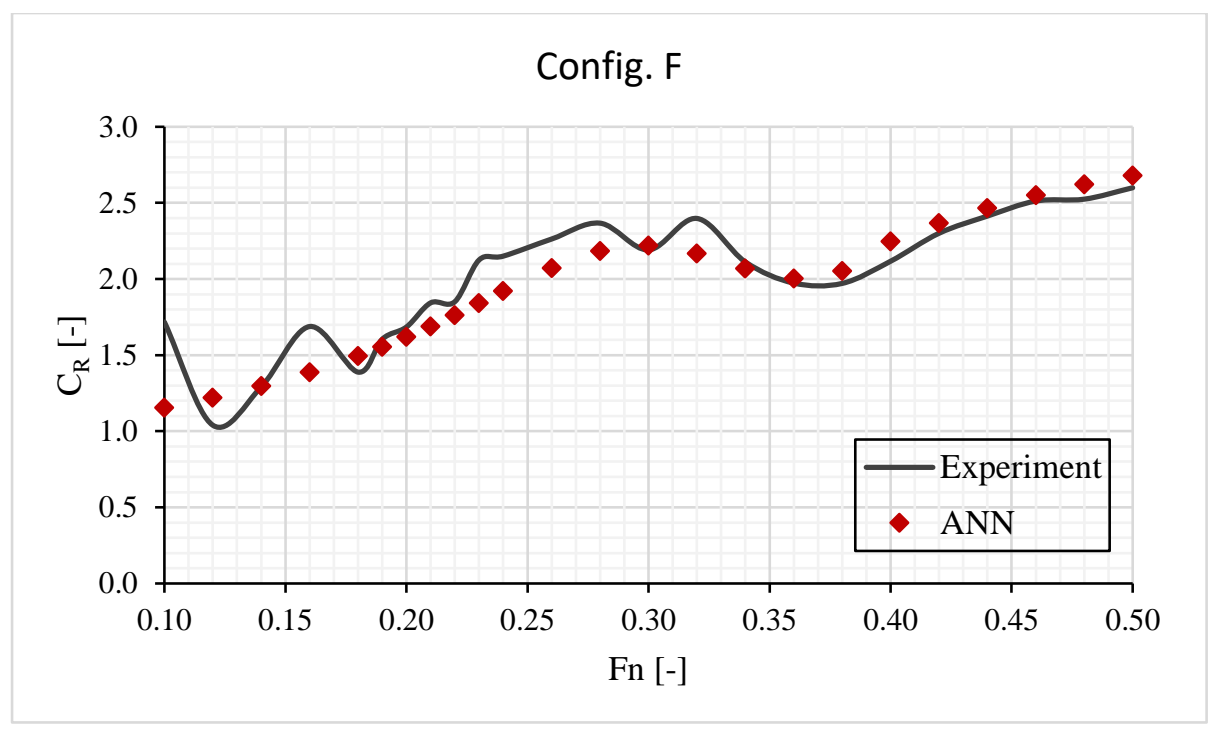

Fig. 9 Comparison of the ANN predictions and experimental results which were not used in the development of the neural network

The relative errors were also calculated to show the accuracy of the neural network by using the following formula:

$$
\varepsilon=\left|\frac{C_{R_{E X P}}-C_{R_{A N N}}}{C_{R_{E X P}}}\right| * 100
$$

The maximum and minimum relative errors were calculated as $32.82 \%$ and $0.51 \%$, respectively, while the mean average value for the relative error was $7.29 \%$. The accuracy of the method can be improved by increasing the data number. However, the neural network results can be used at the preliminary design stage to predict the resistance curve when there is no experimental or simulation data. Carrying out experiments or numerical simulations gives more exact results, but ANN provides a faster prediction option in the preliminary design.

\section{Conclusion}

Determination of the side hull location is of utmost importance during the trimaran design stage. A reasonable resistance reduction can be obtained with careful consideration of side hull positioning which may lead to better performance and less fuel consumption. The location of the side hulls can be determined by carrying out towing tank tests or numerical simulations. Many transverse and longitudinal configurations can be generated, but this is time consuming when trying to find the optimum location. Using an ANN for resistance prediction is faster and easier than traditional methods.

An ANN model for the prediction of the residual resistance coefficient of a trimaran vessel was presented in this study. The developed neural network comprises 4-6-1 neurons in the input layer, hidden layer and output layer, respectively. The input parameters were defined as transverse and longitudinal positions of the side hulls, the longitudinal centre of buoyancy and the Froude number. The experimental data include 8 different configurations of the side hulls with a range of Froude numbers between 0.10 and 0.50 which were used to develop the neural network. One configuration, which was not trained and tested in the developed neural 
network, was used to test the accuracy of the neural network. The results showed that the developed ANN model is able to predict the residual resistance coefficient of the trimaran vessel over a wide speed range. The neural network model captured the complex nature of the trimaran resistance and the results show promising comparisons with experimental results for each configuration. The main hump region of the residual resistance was predicted accurately. The error histogram showed that a large part of the difference between the predicted and actual values was between -0.2 and 0.2 . In addition, the mean average percentage of relative error was $7.29 \%$ for the case study.

The results showed that the ANN is a useful tool to predict the residual resistance coefficient of the trimaran vessel. Using an ANN does not eliminate the need for experiments or numerical simulations since the neural network is developed by using test data. However, it will decrease the number of test case for different configurations and different forward speeds, and can be used to find the optimum side hull location. Furthermore, with more data for different trimaran hull forms, it might be possible to predict the resistance of a given trimaran hull form.

Even though the developed ANN model has good ability to predict the residual resistance of the trimaran vessel, it has some limitations. The developed ANN model does not capture all the trimaran hull forms and can only be used for trimaran hull forms similar to those used in this study. Moreover, the selected side hull configuration should stay in the ranges presented in Table 2 and the forward speed should be in the range of Froude numbers 0.10 to 0.50 .

\section{REFERENCES}

[1] Zhang, J. 1997. Design and hydrodynamic performance of trimaran displacement ships. UCL, 196-213.

[2] Larsson, L., Janson, C.E., Brun P., 1997. A numerical investigation of trimaran configurations. FAST97, Sydney, Australia.

[3] Yang, C., Noblesse, F., Lohner, R., 2001. Practical hydrodynamic optimization of a trimaran. SNAME Transactions. 109, 185-196.

[4] Degiuli, N., Werner, A., Doliner, Z., 2003. Comparison of experimental methods for determining wave pattern resistance of a trimaran based on wave measurements in longitudinal cuts. International shipbuilding progress, 50(1, 2), 57-87.

[5] Doctors, L.J., Sahoo, P.K., 2006. The waves generated by a trimaran. Australian Journal of Mechanical Engineering. 3(2), 183-190. https://doi.org/10.1080/14484846.2006.11464507

[6] Mynard, T., Sahoo, P. K., Mikkelsen, J., McGreer, D. 2008. Numerical and experimental study of wave resistance for trimaran hull forms. 6th International Conference on High-Performance Marine Vehicles, Naples, Italy.

[7] Mahmood, S., De-bo, H., 2011. Resistance calculations of trimaran hull form using computational fluid dynamic. Fourth International Joint Conference on Computational Sciences and Optimization, Kunming, China.

[8] Son, C., Sahoo, P.K., Aribenchi, V., Asapana, S., 2015. CFD simulation of resistance of highspeed trimaran hull forms. 13th International Conference on Fast Sea Transportation, Washington DC, USA. https://doi.org/10.5957/FAST-2015-011

[9] Battistin, D., 2000. Analytical numerical and experimental investigation on the wave resistance interference phenomenon of trimaran configuration. NAV2000 International Conference on Ship and Shipping Research, Venice, Italy.

[10] Mizine, I., Karafiath, G., Queutey, P.,Visonneau, M., 2009. Interference phenomenon in design of trimaran ship. 10th International Conference on Fast Sea Transportation, Athens, Greece.

[11] Zaghi, S., Broglia, R., Di Mascio, A., 2010. Experimental and numerical investigations on fast catamarans interference effects. Journal of Hydrodynamics, Ser. B, 22, 545-549. https://doi.org/10.1016/S10016058(09)60250-X

[12] Farkas, A., Degiuli, N., Martić, I., 2017. Numerical investigation into the interaction of resistance components for a series 60 catamaran. Ocean engineering, 146, 151-169. https://doi.org/10.1016/j.oceaneng.2017.09.043 
[13] Yildiz, B., Sener, B., Duman, S., Datla, R., 2020. A numerical and experimental study on the outrigger positioning of a trimaran hull in terms of resistance. Ocean Engineering, 198, 106938. https://doi.org/10.1016/j.oceaneng.2020.106938

[14] Duman, S., Dogrul, A., Yildiz, B., Datla, R., 2020. Wave interference prediction of a trimaran using form factor. 12th Symposium on High Speed Marine Vehicles, Naples, Italy. https://doi.org/10.3233/PMST200032

[15] Khoob, A.A., Ketabdari, M.J., 2020. Short-Term prediction and analysis of wave-induced motion and load responses of a wave-piercing trimaran. Brodogradnja, 71(2), 123-142. https://doi.org/10.21278/brod71208

[16] Zhang, Y., Wang, P., Liu, Y., Hu, J., 2021. Nonlinear rolling stability and chaos research of trimaran vessel with variable lay-outs in regular and irregular waves under wind load. Brodogradnja, 72(3), 97-123. https://doi.org/10.21278/brod72307

[17] Gong, J., Yunbo, L., Meng, C., Zheng, F., Zhichao, H., 2021. The effect of side-hull position on the seakeeping performance of a trimaran at various headings. Ocean Engineering, 239. https://doi.org/10.1016/j.oceaneng.2021.109897

[18] Jiang, F., Yunbo, L., Jiaye, G., 2021. Study on the manoeuvre characteristics of a trimaran under different layouts by water-jet self-propulsion model test. Applied Ocean Research, 108. https://doi.org/10.1016/j.apor.2021.102550

[19] Ortigosa, I., Lopez, R., Garcia, J., 2009. Prediction of total resistance coefficients using neural networks. Journal of Maritime Research. 6(3), 15-26.

[20] Holtrop, J., Mennen, G.G.J., 1982. An approximate power prediction. International Shipbuilding Progress. 29, 166-170.

[21] Grabowska, K., Szczuko, P., 2015. Ship resistance prediction with artificial neural networks. Signal Processing: Algorithms, Architectures, Arrangements, and Applications (SPA). Poznan, Poland. https://doi.org/10.1109/SPA.2015.7365154

[22] Cepowski, T., 2020. The prediction of ship added resistance at the preliminary design stage by the use of an artificial neural network. Ocean Engineering, 195. https://doi.org/10.1016/j.oceaneng.2019.106657

[23] Yang, Y., Tu, H., Song, L., Chen, L., Xie, D., Sun, J., 2021. Research on accurate prediction of the container ship resistance by RBFNN and other machine learning algorithms. Journal of Marine Science and Engineering, 9(4), 376. https://doi.org/10.3390/jmse9040376

[24] Kim, J.H., Kim, Y., Lu, W., 2021. Prediction of ice resistance for ice-going ships in level ice using artificial neural network technique. Ocean Engineering, 217. https://doi.org/10.1016/j.oceaneng.2020.108031

[25] Royce, R., Mouravieff, A., Zuzick, A., 2011. A trimaran resistance artificial neural network. In Proc. 11th International Conference on Fast Sea Transportation (FAST). Honolulu, Hawaii, USA.

[26] Muk-Pavic, E., Carter, A., McDonald, T., 2013. Resistance prediction using artificial neural networks for preliminary Tri-SWACH design. The International Journal of Maritime Engineering, 156.

[27] Carr, B., Dvorak, R., 2007. Investigation of trimaran interference effects. Unpublished Bachelors Thesis, Webb Institute, Glen Cove, New York, USA.

[28] ITTC, 1957. Report of Resistance Committee. Presented at the Proceedings of 8th ITTC, Madrid, Spain.

[29] Haykin, S., 1999. Neural Networks - A Comprehensive Foundation. Prentice-Hall, second edition, Prentice Hall PTR, Upper Saddle River, NJ, USA.

[30] Margari, V., 2017. Prediction of resistance of MARAD systematic series' hull forms using artificial neural networks. Diploma Thesis, National Technical University of Athens.

[31] Martić, I., Degiuli, N., Majetić, D., Farkas, A. Artificial Neural Network Model for the Evaluation of Added Resistance of Container Ships in Head Waves. Journal of Marine Science and Engineering, 9(8), 826. https://doi.org/10.3390/jmse9080826

[32] The MathWorks Inc, 2018. Neural Network Toolbox. https://www.mathworks.com/products/neuralnetwork.html/ accessed $23^{\text {rd }}$ February 2022.

\footnotetext{
Submitted: $\quad$ 28.12.2021 $\quad$ Burak Y1ld1z, buraky@yildiz.edu.tr Yildiz Technical University, Naval Architecture and Maritime Faculty, Accepted: $\quad$ 22.02.2022. $\quad$ Istanbul, Turkey
} 